\title{
Malaria en niños: relaciones entre nutrición e inmunidad
}

\author{
Silvia Blair, ${ }^{1}$ Jaime Carmona ${ }^{1}$ y Adriana Correa ${ }^{1}$
}

RESUMEN Objetivo. Explorar algunas relaciones entre inmunidad, nutrición y malaria en un grupo de niños de una población con alta incidencia endémica de malaria.

Métodos. Estudio piloto prospectivo y transversal. En 1998, en El Bagre, Antioquia, Colombia, se tomaron consecutivamente 51 niños de uno u otro sexo con sintomas o signos de malaria y gota gruesa positiva, que conformaron el grupo de niños enfermos con malaria. De los hogares infantiles gubernamentales se tomaron aleatoriamente 49 niños sin malaria (ausencia de sintomas y signos, y gota gruesa negativa) que conformaron el grupo de comparación sin malaria. Se calcularon los indices peso/edad, talla/edad y peso/talla; se midieron las concentraciones séricas de albúmina, prealbúmina, apolipoproteína A1 (apoA1), transferrina, cinc, vitamina A, inmunoglobulinas G y M, interleucina 10 (IL-10), factor de necrosis tumoral alfa, interferón gamma, linfocitos y sus poblaciones.

Resultados. De los niños estudiados, $69 \%$ presentaban algún riesgo de desnutrición y $63 \%$ tenían algún riesgo de desnutrición crónica. De las variables inmunitarias y bioquímicas, los enfermos tenían menos apoA1 y albúmina y más IL-10 que los niños sin malaria. Todas las variables bioquímicas mostraron promedios inferiores en el grupo con malaria, riesgo de desnutrición y alteración inmunitaria o bioquímica, mientras que todas las variables inmunitarias presentaron promedios mayores en ese grupo.

Conclusiones. a) La alta frecuencia de desnutrición crónica urge a aplicar medidas de suplementación alimentaria; b) los bajos valores de prealbúmina encontrados pueden deberse a las frecuentes infecciones bacterianas o virales notificadas; c) se observó una grave deficiencia de vitamina $A$, que exige un programa de suplementación; d) se encontró asociación entre los valores bajos de apoA1 y la presencia de malaria, pero no se conoce su secuencia; e) no se observó relación entre los indicadores antropométricos de riesgo de desnutrición y los posibles marcadores bioquímicos del mismo; f) se encontraron altos niveles de IL-10 en los niños enfermos, dato que se notifica por primera vez para Plasmodium vivax.

Palabras clave Malaria, paludismo, desnutrición, índices antropométricos, apolipoproteína A1, inmunidad, interleucina 10.

En el departamento de Antioquia, como en Colombia y el mundo, la ma-

\footnotetext{
1 La correspondencia debe dirigirse a Silvia Blair a la siguiente dirección postal: Grupo Malaria. Carrera $51 \mathrm{D} \mathrm{N}^{\circ}$ 62-29, Laboratorio de Malaria, Facultad de Medicina, Universidad de Antioquia, Medellín, Colombia. Teléfonos: (574) 5106058/ 5106061. Correo electrónico: sblair@ catios.udea. edu.co
}

laria es un grave problema de salud pública (1-4). Por otra parte, en Colombia, a pesar del subregistro, la desnutrición es responsable del $25 \%$ de las muertes en niños (5). En el Bajo Cauca antioqueño, donde se realizó el presente trabajo, la desnutrición proteinico-calórica ocupó el décimo lugar entre las primeras causas de muerte en 1997 (4).

Hay una relación estrecha entre el estado nutricional, el funcionamiento inmunitario y la susceptibilidad a las infecciones. Estas últimas se incrementan cuando la desnutrición es grave, ya sea de tipo general o específica para 
algún nutriente (6-13), aunque algunos afirman que la desnutrición protege contra la malaria o sus complicaciones $(14,15)$. La desnutrición es la causa más frecuente de inmunodeficiencia infantil en el mundo, aumenta la frecuencia y gravedad de las infecciones y sus efectos son más perniciosos sobre la inmunidad celular (16).

A pesar de la alta frecuencia y la gravedad de la desnutrición en todo el mundo, en particular entre los niños, la evaluación del fenómeno continúa atada a las antiguas mediciones antropométricas, sin que, por ejemplo, se haya podido avanzar en la consolidación de un sistema de evaluación nutricional basado en mediciones bioquímicas o en un sistema antropométrico y bioquímico. Tradicionalmente, para clasificar el riesgo de desnutrición se elaboran índices antropométricos basados en el sexo, la edad, el peso y la talla del niño, tales como el peso para la edad $(\mathrm{P} / \mathrm{E})$, la talla para la edad $(\mathrm{T} / \mathrm{E})$ y el peso para la talla $(\mathrm{P} / \mathrm{T})(17)$. La prealbúmina puede reflejar mejor a corto plazo los cambios sucedidos en el metabolismo de las proteínas. También los niveles sanguíneos de albúmina, transferrina, apolipoproteína A1 (apoA1) y retinol (vitamina A) se han empleado para explorar el estado nutricional (18-20). Hall propuso un índice de desnutrición en función de parámetros antropométricos y bioquímicos, considerando dentro de estos la albúmina y la prealbúmina, y lo llamó índice pronóstico inflamatorio y nutricional (18). Sin embargo, la evaluación estándar del estado y del riesgo nutricionales descansa todavía en los criterios antropométricos.

Por su parte, la evaluación del estado inmunitario incluye la medición de inmunoglobulinas $G$ y $M$ (IgG e IgM, respectivamente); citocinas, como las interleucinas (IL), los interferones (IFN) y los factores de necrosis tumoral (FNT); y también conteos de células sanguíneas, como leucocitos totales, linfocitos y sus subpoblaciones.

En la enfermedad aguda por Plasmodium falciparum se han detectado niveles séricos elevados de varias citocinas, como las IL-1, IL-6, IL-8 e IL-10, el
FNT $\alpha$ y el INF $\gamma$ (21-23). Las concentraciones altas de FNT $\alpha$ han sido asociadas con las complicaciones cerebrales de la malaria (24), pero también se han presentado en la enfermedad no complicada y, al parecer, están más relacionadas con la parasitemia y la fiebre que con la gravedad misma de la enfermedad $(25,26)$. Parece que la IL10 modula inmunológicamente la producción de interferón por las células TCD4 ${ }^{+}$tipo TH1, inhibe la producción de citocinas - como el FNT $\alpha$ - y puede estar involucrada en la patogenia de la malaria $(27,28)$.

El objetivo del presente trabajo fue estudiar las relaciones entre un grupo de variables indicadoras del estado inmunitario y nutricional (tanto antropométricas como bioquímicas) en dos grupos de niños, uno con malaria y otro sin ella.

\section{MATERIALES Y MÉTODOS}

\section{Clase de estudio}

Se aplicó un diseño prospectivo y transversal. El estudio fue piloto (exploratorio), porque no había información sobre la población en estudio que permitiese una mejor aproximación.

\section{Diseño y selección de la muestra poblacional}

En el municipio de El Bagre, situado en la región del Bajo Cauca, al noreste del departamento de Antioquia, Colombia, se estudiaron dos grupos de niños de ambos sexos. El primero estuvo conformado por 51 enfermos de malaria, con edades entre 4 y 9 años, mientras que el segundo incluyó 49 niños sin malaria, en el mismo intervalo de edades que los anteriores. Se tomó ese tamaño de muestra porque, según el análisis inicial, permitía realizar las comparaciones estadísticas más importantes.

El grupo de enfermos se conformó por los primeros 51 pacientes con malaria, comprobada según el examen de gota gruesa, que acudieron por su propia iniciativa al puesto de malaria del hospital de El Bagre y que cumplían con los requisitos de inclusión expuestos más adelante. Para conformar el grupo de niños sin malaria se tomaron al azar 49 niños procedentes tanto de zonas urbanas como rurales de este municipio, que asistían a alguna de las escuelas (de 7 a 9 años de edad) o a los hogares infantiles patrocinados por el Instituto Colombiano de Bienestar Familiar (ICBF) (de 4 a 6 años). La selección aleatoria se hizo entre aquellos que no presentaban síntomas de malaria y eran negativos al examen de gota gruesa. Ningún niño estaba institucionalizado, es decir, todos vivían en sus hogares y asistían solo durante parte del día a las escuelas u hogares.

\section{Evaluación del riesgo de desnutrición}

Se registró la edad, el peso, la talla y el sexo de cada niño estudiado. Se usó el módulo Epinut, del programa Epi Info v. 6.04, para analizar los datos de la evaluación antropométrica (29). Este programa toma los valores observados de sexo, edad, peso y talla y calcula los índices antropométricos $\mathrm{P} / \mathrm{E}$ (que refleja la desnutrición global), $\mathrm{T} / \mathrm{E}$ (la desnutrición crónica) y $\mathrm{P} / \mathrm{T}$ (para la desnutrición aguda) $(30,31)$. Los índices $\mathrm{P} / \mathrm{T}$ y $\mathrm{T} / \mathrm{E}$ son los más frecuentemente empleados para determinar el estado nutricional, pues permiten distinguir entre diferentes procesos fisiológicos y biológicos $(30,31)$. En estudios transversales como el presente, en los que solo se hace una medición antropométrica, no es posible obtener un diagnóstico del estado nutricional, pero sí una clasificación del riesgo de desnutrición en dependencia de los valores de los tres índices antropométricos de cada niño, según el porcentaje de adecuación a la mediana (cuadro 1) y las unidades Z (cuadro 2). Los valores de la escala en unidades $Z$ son los propuestos por Epinut-Epi Info (29), mientras que los valores del porcentaje de adecuación a la mediana se definieron, como se recomienda, a partir de los datos observados (29). 
CUADRO 1. Categorías empleadas para expresar el resultado final del análisis del riesgo de desnutrición de cada niño, según el porcentaje de adecuación a la mediana. El Bagre, Antioquia, Colombia, 1998

\begin{tabular}{lccc}
\hline & \multicolumn{3}{c}{ Intervalos } \\
\cline { 2 - 4 } Categoría & $\mathrm{P} / \mathrm{E}$ & $\mathrm{P} / \mathrm{T}$ & $\mathrm{T} / \mathrm{E}$ \\
\hline Riesgo alto & $<70$ & $<78$ & $<85$ \\
Riesgo medio & $70,0-79,99$ & $78,0-85,99$ & $85,0-89,99$ \\
Riesgo bajo & $80,0-89,99$ & $86,0-91,99$ & $90,0-89,99$ \\
Normal & $90,0-109,99$ & $92,0-107,99$ & $90,0-94,99$ \\
Exceso & 110,0 o más & 108,0 o más & 95,0 o más \\
\hline
\end{tabular}

CUADRO 2. Categorías empleadas para expresar el resultado final del análisis del riesgo de desnutrición de cada niño, según las unidades $\mathbf{Z}$ (expresadas en desviacioBagre, Antioquia, Colombia, 1998

\begin{tabular}{lc}
\hline Categoría & Intervalos \\
\hline Riesgo alto & $<-3,01$ \\
Riesgo medio & de $-3,0$ a $-2,01$ \\
Riesgo bajo & de $-2,0$ a $-1,01$ \\
Normal & de $-1,0$ a 1,99 \\
Exceso & 2,0 o más \\
\hline
\end{tabular}

\section{Evaluación bioquímica}

Se estudiaron las variables bioquímicas de 25 individuos de cada grupo. nes estándar con respecto al promedio). El
La prealbúmina, la apoA1 y la transferrina se cuantificaron por nefelometría, mediante estuches PAB, APOA y TRF, respectivamente, (Beckman Coulter, Inc., Alemania); la albúmina se evaluó por espectrofotometría, con un estuche Albumine (Abbot Laboratories, EE.UU.); los de cinc por espectroscopia de absorción atómica en el Laboratorio de Productos Naturales de la Facultad de Química de la Universidad de Antioquia. Los niveles de vitamina A se establecieron mediante un método desarrollado y aplicado en el Laboratorio de Química Farmacéutica de la Universidad de Antioquia: la vitamina A como éster se convirtió a vitamina A como alcohol por vía de hidrólisis alcalina; luego se extrajo de la mezcla saponificada; el extracto fue purificado por cromatografía en fase
CUADRO 3. Valores de referencia empleados para la evaluación bioquímica. El Bagre, Antioquia, Colombia, 1998

\begin{tabular}{lcc}
\hline \multicolumn{1}{c}{ Variable } & Valores de referencia & Fuente $^{a}$ \\
\hline Prealbúmina & $18,0-44,5 \mathrm{mg} / \mathrm{dL}$ & $(1)$ \\
Albúmina & $300-5200 \mathrm{mg} / \mathrm{dL}$ & $(1)$ \\
Apolipoproteína A1 & $94-178 \mathrm{mg} / \mathrm{dL}$ en niños & $(1)$ \\
& $101-198 \mathrm{mg} / \mathrm{dL}$ en niñas & $(1)$ \\
Transferrina & $211,6-360,3 \mathrm{mg} / \mathrm{dL}$ & $(2)$ \\
Cinc & $0,5-1,2 \mathrm{mg} / \mathrm{dL}$ & $(3)$ \\
Vitamina A & $0,3-0,8 \mathrm{fg} / \mathrm{dL}$ & \\
\hline a 1 Laboratorio Clínico de la Congregación Mariana: según estudio de la población de Medellín. \\
2 Laboratorio de Productos Naturales, Universidad de Antioquia: basados en personas aparentemente \\
sanas de la ciudad de Medellín. \\
3 Laboratorio Química Farmacéutica, Universidad de Antioquia: basados en personas aparentemente \\
sanas de la ciudad de Medellín.
\end{tabular}

\section{Cuantificación de la población linfoide}

Se cuantificó la población linfoide de 20 niños con malaria y 26 sin malaria mediante citometría de flujo, con lectura final en un Fac Sort ${ }^{\circledR}$ (Benton Dickinson, EE.UU.), en la unidad de citometría de la Facultad de Medicina de la Universidad de Antioquia. Los valores (células $/ \mathrm{mm}^{3}$ ) considerados pos de todos los casos estudiados. La IgG se midió por inmunofluorescencia indirecta (IFI) (32) y la IgM megeno se emplearon esquizontes de $P$. falciparum, cepa FCB-2, obtenidos según el procedimiento descrito por Lambros y Vanderberg (33). Se consi1:16 o mayores, y para la IgM 1:32 o mayores. Las técnicas IFI y ELISA empleadas detectan anticuerpos contra cualquier especie de Plasmodium. 
como normales fueron los propuestos por Commans-Bitter y colaboradores (34): linfocitos totales: de 1100 a 6 900; CD3: de 700 a 4 500; CD4: de 300 a 2 400; CD8: de 300 a 1 800; CD4/CD8: de 0,9 a 2,9. Los leucocitos totales se tomaron como expresión de leucocitosis cuando fueron mayores de 10000 $\mathrm{cel} / \mathrm{mm}^{3}$.

\section{Evaluación de la respuesta linfoproliferativa}

Las respuestas policlonal y oligoclonal se determinaron mediante la confrontación de las células con un preparado de fitohemaglutinina y con el mismo extracto antigénico de $P$. falciparum, cepa FCB-2, descrito para la medición de anticuerpos IgG e IgM (35).

\section{Evaluación parasitológica}

La evaluación parasitológica practicada a todos los niños de ambos grupos se realizó mediante gota gruesa y frotis de sangre periférica. Se hizo el diagnóstico de especie de Plasmodium y el conteo de parásitos (36).

\section{Evaluación clínica}

Todos los niños fueron evaluados por un médico para conocer la presencia de síntomas o signos compatibles con malaria, definida como la presencia de uno o varios de esos síntomas o signos y un examen de gota gruesa que demostrara la presencia de parásitos del género Plasmodium.

\section{Criterios de inclusión en el estudio}

a) Residir en el municipio de El Bagre, bien sea en área rural o urbana; b) tener entre 4 y 9 años de edad; c) acudir espontáneamente al puesto de diagnóstico con malaria, o estar vinculado a alguna escuela o uno de los hogares infantiles patrocinados por el ICBF; d) aceptar participar en el estudio, mediante consentimiento infor- mado y escrito firmado por los padres o acudientes.

\section{Criterios de exclusión del estudio}

Presencia, en el momento de ingreso al estudio, de alguna enfermedad distinta a la malaria, según evaluación clínica (entrevista al acudiente y examen clínico del niño) realizada por los investigadores.

\section{Estrategia para el análisis de los datos}

Primero se examinó si las variables epidemiológicas de los dos grupos eran comparables (distribuciones de frecuencia y medidas descriptivas). Para evaluar la asociación estadística entre variables no métricas se usaron las pruebas de $\chi^{2}$, de Fisher y la razón de posibilidades de Mantel-Haenszel. La prueba $t$ o la prueba $\mathrm{F}$ se usaron para comparar promedios $\mathrm{y}$, cuando no se cumplían los requisitos para usar estas pruebas, se empleó la prueba no paramétrica de Kruskal-Wallis para comparar rangos $(29,37,38)$.

El segundo paso del análisis consistió en comparar el grupo "enfermos con riesgo de desnutrición" con el grupo "sin malaria y sin riesgo de desnutrición" en términos bioquímicos e inmunitarios. Este análisis no reflejó diferencias significativas, excepto para las variables apoA1, albúmina, anticuerpos, IL-10 y leucocitos $(P<0,05)$. Este análisis no se incluye en el artículo.

En el tercer paso se conformaron dos grupos extremos: uno con aquellos niños que padecían malaria y presentaban riesgo de desnutrición, y otro con los que no presentaban ninguno de los dos fenómenos. Para la comparación de las variables bioquímicas e inmunológicas, donde sí se observaron diferencias significativas, se emplearon la prueba de Students, el análisis de varianza (prueba F) y el procedimiento de comparaciones múltiples según Newman y Keuls, usados por SGPlus v. $7.1(29,37,38)$. Para el análisis estadístico se usó además el programa Epi Info v. 6.04 (29).

\section{RESULTADOS}

Los grupos de niños enfermos con malaria y sin malaria fueron comparables debido a su similitud en cuanto a la proporción por sexos, edades, tiempo de permanencia en la zona, frecuencia de riesgo de desnutrición, antecedentes de otras enfermedades infecciosas y asistencia a algún programa de apoyo nutricional de los existentes en el municipio (hogares del ICBF). Entre los dos grupos hubo diferencia significativa $(P<0,0001)$ en los antecedentes de infecciones de malaria en el año anterior al estudio (70\% en los enfermos y $10 \%$ en los del otro grupo) y en el promedio de episodios palúdicos por año (2,01 en los enfermos y 0,18 en los otros).

Entre los niños con malaria no hubo ninguno con enfermedad complicada; $66 \%$ de las infecciones fueron producidas por P. vivax, $30 \%$ por P. falciparum y $4 \%$ por ambas especies (infección mixta). La parasitemia promedio en los pacientes con $P$. vivax fue de 5495 parásitos $/ \mathrm{mm}^{3}$, mientras que en la infección por P. falciparum fue de 3820 parásitos $/ \mathrm{mm}^{3}$. En general, $70 \%$ de los pacientes presentó parasitemias de 5000 parásitos $/ \mathrm{mm}^{3}$ o menos, y solo 10\% tuvo más de 20000 parásitos/ $\mathrm{mm}^{3}$.

\section{Riesgo de desnutrición}

El cuadro 4 presenta el resultado de la clasificación antropométrica de los niños estudiados. Para cada índice, el riesgo de desnutrición es similar en los niños con y sin malaria $(P>0,10)$. En conjunto, 63\% tuvo algún riesgo de desnutrición crónica, 7\% mostró algún riesgo de desnutrición aguda y $49 \%$ presentó algún riesgo de desnutrición global. Si se considera en riesgo de desnutrición a quien presentara algún riesgo al menos en alguno de los tres índices, 39\% (60/153) de los niños con malaria y $40 \%(59 / 147)$ de los niños 
CUADRO 4. Resultados de la evaluación antropométrica con tres índices, de acuerdo con los puntajes $Z$ y según la presencia de malaria. El Bagre, Antioquia, Colombia, 1998

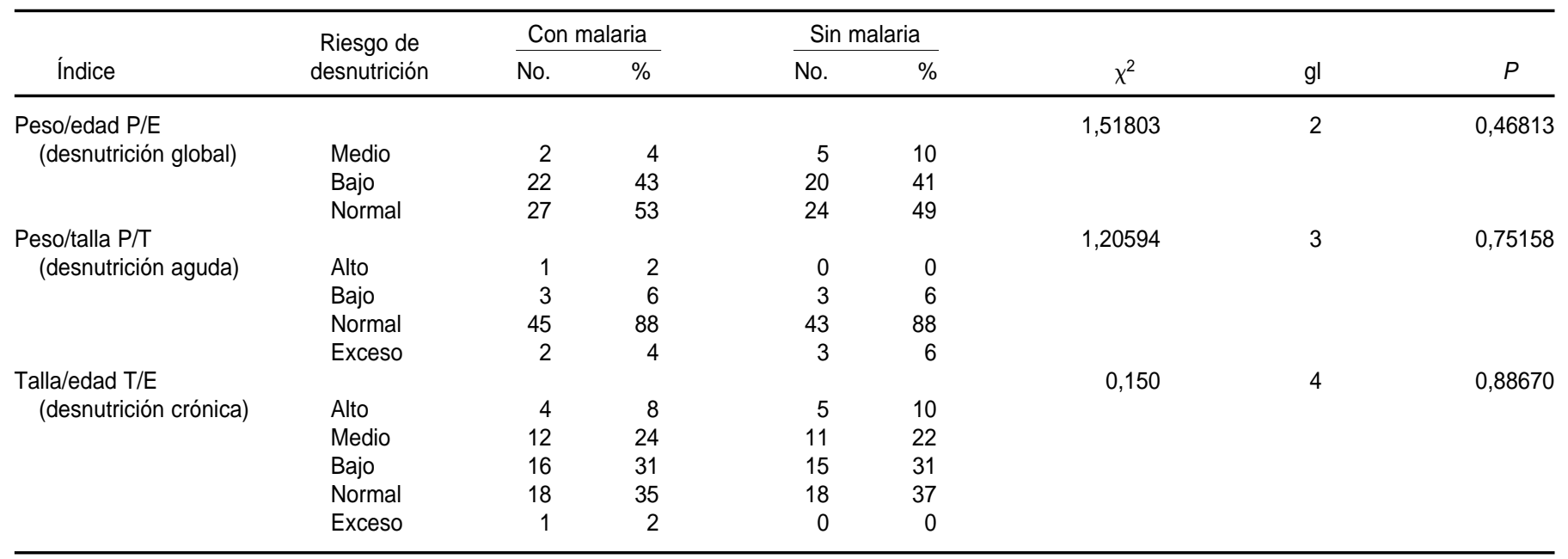

del grupo sin la enfermedad tenían ese riesgo.

Se debe subrayar que no se halló diferencia entre los grupos según la asistencia o no a algún programa de apoyo nutricional de los existentes en el municipio (hogares del ICBF). El riesgo de desnutrición no mostró diferencias cuando se analizó en función del tiempo de permanencia en el municipio, el número de episodios de malaria sufridos en el último año, el número de personas que conviven con el niño o el número de menores de 15 años que conviven con el niño $(P>0,05)$.

\section{Evaluación de las variables bioquímicas}

Como se observa en el cuadro 5, todos los promedios fueron mayores en los niños sin malaria que en los que presentaban la enfermedad, pero esta diferencia fue estadísticamente significativa solo en los casos de albúmina y apoA1, esta última tanto en niños como en niñas.

a) Albúmina: al menos $96 \%$ de ambos grupos tuvieron cifras normales de albúmina. El promedio de albúmina en los enfermos de malaria fue de $4068 \mathrm{mg} / \mathrm{dL}$ mientras que en aquellos sin la enfermedad fue de 4431 $\mathrm{mg} / \mathrm{dL}(t=4,62042 ; P=0,000028)$.

b) ApoA1: mantuvo valores bajos (menores que los normales) en $81 \%$ de los niños enfermos $(21 / 26)$ y en solo $39 \%$ de los que no tenían la enfermedad (9/23); en las niñas, $92 \%(23 / 25)$ de las enfermas tuvieron cifras bajas, frente a $63 \%(10 / 16)$ de las no enfermas. El promedio de apoA1 en los niños con malaria fue $68,03 \mathrm{mg} / \mathrm{dL}$ y de $99,03 \mathrm{mg} / \mathrm{dL}$ en los que no tenían malaria, mientras que en las niñas enfermas fue 70,53 $\mathrm{mg} / \mathrm{dL}$ contra $102,38 \mathrm{mg} / \mathrm{dL}$ en aquellas en que la enfermedad estaba ausente. Tanto en niños como en niñas se observó una fuerte asociación entre las variables malaria (sí/no) y el nivel de apoA1 (bajo/normal). En los niños y niñas, tanto con riesgo de desnutrición como sin este riesgo, la presencia de la malaria redundó en una diferencia significativa en el promedio de apoA1, en comparación con aquellos que no tenían malaria, quienes presentaron cifras superiores.

Aunque no difirieron estadísticamente entre sí, como indica el cuadro 5, en ambos grupos hubo muchos niños con niveles de prealbúmina y vitamina A muy bajos con respecto al valor normal, mientras que los valores de trans- ferrina y cinc fueron normales en casi todos los niños de ambos grupos.

a) Prealbúmina: se mantuvo baja en $92 \%$ de los enfermos y en $81 \%$ de los no enfermos. Los promedios fueron 13,4 y $14,8 \mathrm{md} / \mathrm{dL}$ en las personas con y sin malaria, respectivamente $(P>0,05)$.

b) Vitamina A: $65 \%$ de los pacientes con malaria y $35 \%$ de los niños sin la enfermedad tuvieron valores bajos; los promedios en enfermos y no enfermos fueron de 0,14 y $0,22 \mathrm{fg} / \mathrm{mL}$, respectivamente $(P>0,05)$.

c) Transferrina: $68 \%$ de los enfermos con malaria y $73 \%$ de los no enfermos tuvieron cifras normales, con promedios de 232,1 y 232,6 mg/dL ( $P>0,05)$.

d) Cinc: $76 \%$ de los niños con malaria y $92 \%$ de los que no la tenían presentaron cifras normales, y los promedios globales fueron 0,78 y $0,89 \mathrm{mg} /$ $\mathrm{dL}$, respectivamente $(P>0,05)$.

\section{Evaluación de las variables inmunológicas}

Todas las variables inmunitarias tuvieron promedios superiores en los niños con malaria, aunque los dos grupos fueron estadísticamente similares en cuanto a FNT $\alpha$, leucocitos, linfocitos totales, linfocitos CD3, CD4, CD8 y 
CUADRO 5. Promedio $(\bar{X})$ y desviación estándar $(D E)$ de las variables bioquímicas e inmunitarias en niños maláricos y sin malaria. El Bagre, Antioquia, Colombia, 1998

\begin{tabular}{|c|c|c|c|c|c|c|c|c|}
\hline \multirow[b]{2}{*}{ Variables (mg/dL) } & \multicolumn{3}{|c|}{ Niños con malaria } & \multicolumn{3}{|c|}{ Niños sin malaria } & \multirow[b]{2}{*}{$t$} & \multirow[b]{2}{*}{$P$} \\
\hline & No. & $\bar{x}$ & $D E$ & No. & $\bar{x}$ & $D E$ & & \\
\hline \multicolumn{9}{|l|}{ Bioquímicas } \\
\hline ApoA1 niñas & 25 & 71 & 24 & 26 & 102 & 14 & 5,72926 & $0,8 \times 10^{-8}$ \\
\hline Prealbúmina & 25 & 13 & 4 & 26 & 15 & 3 & 1,45119 & 0,153101 \\
\hline Albúmina & 25 & 4,068 & 341 & 26 & 4,431 & 205 & 4,62042 & 0,000028 \\
\hline Transferrina & 25 & 232 & 44 & 26 & 233 & 35 & 0,0444 & 0,964765 \\
\hline \multicolumn{9}{|l|}{ Inmunitarias } \\
\hline Leucocitos $/ \mathrm{mm}^{3}$ & 20 & 9,017 & 3,034 & 26 & 9,318 & 4,181 & 0,27139 & 0,787357 \\
\hline Linfocitos $/ \mathrm{mm}^{3}$ & 20 & 3,667 & 1,395 & 26 & 4,435 & 1,626 & 1,68666 & 0,098747 \\
\hline $\mathrm{CD} 3 / \mathrm{mm}^{3}$ & 20 & 2,383 & 966 & 26 & 2,817 & 1,122 & 1,37976 & 0,174634 \\
\hline $\mathrm{CD} 4 / \mathrm{mm}^{3}$ & 20 & 1,350 & 563 & 26 & 1,674 & 789 & 1,55515 & 0,127074 \\
\hline $\mathrm{CD} 8 / \mathrm{mm}^{3}$ & 20 & 817 & 344 & 26 & 910 & 413 & 0,81498 & 0,419475 \\
\hline IL $10(\mathrm{pg} / \mathrm{ml})$ & 51 & 266 & 342 & 49 & 8 & 8 & 5,27033 & $8,1 \times 10^{-7}$ \\
\hline
\end{tabular}

a Títulos positivos de $\lg G \geq 1: 16$ sin diferencia significativa entre los dos grupos.

${ }^{b} T$ ítulos positivos de $\lg \mathrm{M} \geq 1: 32$ sin diferencia significativa entre los dos grupos.

relación CD4/CD8, y solo mostraron diferencia en los promedios de IL-10 e IgM (cuadro 5).

El título medio de IgG en el grupo con malaria fue 1:324, mientras que en el otro fue 1:5 $(P>0,05)$. La IgG fue positiva ( $\geq 1: 16$ ) en $57 \%$ de los enfermos (29 casos) frente a solo $8 \%$ de los no enfermos (4 niños), sin diferencia significativa entre los promedios generales de los dos grupos $(P>0.05)$. La IgM tuvo un título medio de 1:51 en el grupo de niños con malaria y de 1:7 en el otro $(P<0,05)$, y fue positiva $(\geq 1: 32)$ en $39 \%$ de los enfermos (20 casos) y en $4 \%$ de los niños sin la enfermedad ( 2 casos), aunque esta diferencia no fue estadísticamente significativa $(P>0,05)$.

En el grupo con malaria, las variables inmunitarias se comportaron de forma similar, independientemente de la especie parasitaria.

El FNT $\alpha$ se mantuvo normal en $82 \%$ de los niños con malaria y en $88 \%$ del otro grupo, y no hubo diferencia significativa entre los promedios: 13,9 y $18,8 \mathrm{pg} / \mathrm{mL}$, con desviaciones estándar de 55,0 y $67,3 \mathrm{pg} / \mathrm{mL}$, respectivamente $(P>0,05)$.
Los valores de IL-10 fueron altos en $96 \%$ de los enfermos con malaria y en $61 \%$ de los que no tenían la enfermedad, con diferencia significativa entre sus promedios $(266,2 \mathrm{pg} / \mathrm{mL}$ vs. 8,5 $\mathrm{pg} / \mathrm{mL}$ y desviaciones típicas de 342,0 y $8,2 \mathrm{pg} / \mathrm{mL}$, respectivamente; $t=$ 5,$27033 ; P=8,1 \times 10^{-7}$ ) (cuadro 5). En los enfermos, los títulos altos de IL-10 predominaron entre quienes tenían riesgo de desnutrición (33 de 42 niños), con una asociación significativa entre el riesgo de desnutrición (si/no) y el nivel de IL-10 (alto/normal). En cambio, entre los no enfermos, estas dos variables no presentaron asociación (Fisher de 2 colas, $P=0,66845$ ), y los títulos bajos fueron mucho más frecuentes que los altos. El cuadro 6 presenta los promedios y las desviaciones estándar de IL-10 en siete grupos conformados según la presencia de malaria y el riesgo de desnutrición. El análisis de estos datos permite afirmar que el promedio de IL-10 mostró diferencia significativa solo entre enfermos y no enfermos, independientemente del riesgo de desnutrición, por lo que puede concluirse que este no influyó.
Variables bioquímicas e inmunitarias según el riesgo de desnutrición

Al comparar los grupos "con riesgo de desnutrición" $(n=69)$ y "sin riesgo de desnutrición" ( $n=31)$, independientemente de la presencia de malaria en cada uno de ellos, ninguna de las variables bioquímicas o inmunitarias (excepto el conteo de leucocitos) mostró diferencia significativa $(P>0,05)$.

De las variables bioquímicas o inmunitarias entre los grupos "con malaria con riesgo de desnutrición" y "sin malaria sin riesgo de desnutrición", hubo diferencias significativas en apoA1 (en niños y niñas), albúmina e IL-10.

\section{Comparación de los grupos extremos}

Se compararon dos grupos extremos: niños (de uno $\mathrm{u}$ otro sexo) con malaria, con riesgo de desnutrición y con alteración inmunitaria o bioquímica, y niños (también de cualquier sexo) sin malaria, sin riesgo de desnu- 
CUADRO 6. Niveles de IL-10 (pg/mL) en los grupos comparados, según la presencia o ausencia de malaria (M+, M-) y el riesgo de desnutrición (D+, D-) y la especie parasitaria (P.f.: Plasmodium falciparum; P.v.: Plasmodium vivax). El Bagre, Antioquia, Colombia, 1998

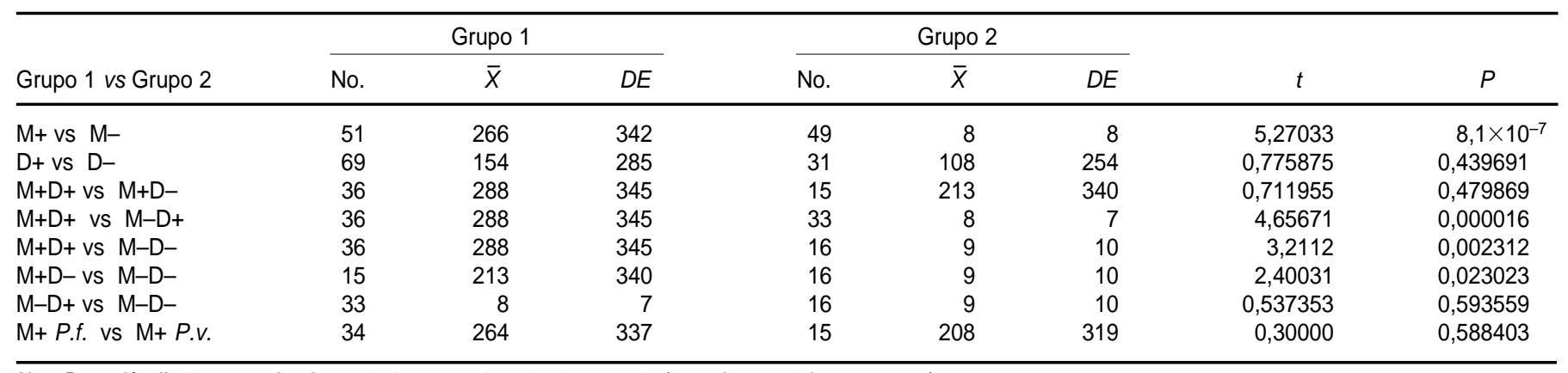

Nota: Regresión: IL-10 en parasitemia, $r=0,54$ con $n=51 ; r=0,73$ con $n=47$ (se excluyeron 4 datos extremos).

trición y sin alteración inmunitaria o bioquímica $\mathrm{O}$, en otros términos, el grupo más afectado frente al grupo normal que no presentaba ninguno de esos factores. Todas las variables bioquímicas e inmunitarias presentaron diferencias significativas, con excepción de los títulos de IgG (cuadro 7). En comparación con el grupo normal, todas las variables bioquímicas del grupo más afectado mostraron promedios muy inferiores, mientras que los promedios de todas las variables inmunitarias fueron mayores.

\section{DISCUSIÓN}

Los dos grupos (enfermos y no enfermos) fueron comparables según sus condiciones de vida, edad, sexo, tiempo de permanencia en la zona, y antecedentes de infecciones, entre otros parámetros, pero difirieron por sus antecedentes de malaria. Las diferencias que se hallaron entre los dos grupos podrían deberse, en consecuencia, a la existencia de malaria en el momento del estudio, o a los antecedentes de la enfermedad, más frecuentes en el grupo de enfermos.

El riesgo siete veces mayor de volver a presentar malaria que se observó en los niños que presentaban la enfermedad es un elemento que llama la atención y que podría explicarse por diferencias genéticas, por peculiaridades en el comportamiento de los indi- viduos o por recaídas debidas a $P$. vivax, pero los resultados obtenidos no permiten explicar estas diferencias según el riesgo de desnutrición ni por las variables inmunitarias o bioquímicas estudiadas.

Ambos grupos presentaron una alta frecuencia de riesgo de desnutrición global y, lo que es más significativo, de desnutrición crónica, que refleja una privación alimentaria muy prolongada, derivada de sus precarias condiciones de vida y epidemiológicas, similares para ambos grupos. Esta ausencia de diferencia en cuanto al riesgo de desnutrición entre los dos grupos fue comprobada, a pesar de que el grupo de niños con malaria tenía significativamente más antecedentes de la enfermedad, lo que podría hacer pensar que su estado pudiera haber estado más afectado, aunque esto no fue comprobado.

Por otra parte, los bajos valores de prealbúmina y de apoA1 en la mayoría de estos niños sugieren que recientemente habían ocurrido cambios en el metabolismo de las proteínas $(19,39)$, como los asociados a la desnutrición aguda inducida por los episodios frecuentes de otras infecciones bacterianas o virales, que se hallaron con una frecuencia similar en ambos grupos. A esto se debe añadir la influencia de la malaria que sufría uno de los grupos en el momento del estudio y que podría explicar por qué los niños de ese grupo presentaban valores más bajos de pre- albúmina y, sobre todo, de apoA1. Otro elemento que refuerza esta hipótesis fue la baja cantidad de vitamina A hallada en los dos grupos, la cual es un antioxidante con alta demanda en los procesos estresantes —como los debidos a las infecciones-, por lo que resultó interesante observar que los niños con malaria tenían cifras mucho más bajas que aquellos que no tenían la enfermedad, lo cual podría explicarse por el episodio de malaria que sufrían en ese momento. Las cifras de vitamina $\mathrm{A}$ encontradas fueron tan bajas que obligan a proponer que se instaure de inmediato un programa de suplemento de esta vitamina, conforme a las recomendaciones de la Organización Mundial de la Salud $(40,41)$.

Se encontró una asociación significativa entre los valores bajos de apoA1 y la presencia de malaria. Es posible que la malaria reduzca la concentración de apoA1 o que ésta sea expresión de un estado nutricional deteriorado que predisponga al desarrollo de la malaria, ya que los niños bien nutridos, tanto los que tenían la enfermedad como los que no la padecían, presentaron con más frecuencia niveles normales de apoA1 que aquellos con desnutrición.

Al evaluar la influencia que la malaria, el riesgo de desnutrición o ambos ejercieron sobre las variables bioquímicas e inmunitarias, se observó que, individualmente, la malaria actuó de tal manera que los niños enfermos tuvieron, en general, cifras diferentes de las 
CUADRO 7. Valores de las variables bioquímicas e inmunitarias en niños con malaria, con riesgo de desnutrición y con alteración inmunitaria o bioquímica (grupo más afectado) y en niños sin malaria, sin riesgo de desnutrición y sin alteración inmunitaria o bioquímica (grupo normal). El Bagre (Antioquia), 1998

\begin{tabular}{|c|c|c|c|c|c|c|c|c|}
\hline \multirow[b]{2}{*}{ Variable $^{a}$} & \multicolumn{3}{|c|}{ Grupo más afectadob } & \multicolumn{3}{|c|}{ Grupo normal ${ }^{b}$} & \multirow[b]{2}{*}{$t$} & \multirow[b]{2}{*}{$P$} \\
\hline & No. & $\bar{x}$ & $D E$ & No. & $\bar{x}$ & $D E$ & & \\
\hline \multicolumn{9}{|l|}{ Bioquímicas } \\
\hline ApoA1 mujeres & 17 & 65 & 21 & 5 & 105 & 6 & 4,26449 & 0,000379 \\
\hline Prealbúmina & 19 & 13 & 3 & 3 & 21 & 0,85 & 4,73061 & 0,000128 \\
\hline Albúmina & No hay casos ${ }^{c}$ & & & & & & & \\
\hline \multicolumn{9}{|l|}{ Inmunitarias } \\
\hline Leucocitos & 6 & 12,439 & 2,694 & 8 & 6,277 & 1,215 & 5,7886 & 0,000086 \\
\hline Linfocitos $^{c}$ & No hay $\operatorname{casos}^{c}$ & & & & & & & \\
\hline $\lg G(I F I)$ & 17 & 262 & 487 & 13 & 0 & $0^{d}$ & 2,22 & 0,155915 \\
\hline IgM (Elisa) & 10 & 203 & 174 & 15 & 3 & 5 & 4,48543 & 0,000168 \\
\hline FNT $\alpha$ & 3 & 192 & 139 & 14 & 2 & 6 & 5,87464 & 0,000030 \\
\hline
\end{tabular}

a Unidades de variables bioquímicas: $\mathrm{mg} / \mathrm{dL}$ (vitamina $\mathrm{A}$ : $\mathrm{fg} / \mathrm{dL}$ ); las variables inmunitarias: células $/ \mathrm{mm}^{3}$ o $\mathrm{pg} / \mathrm{mL}$.

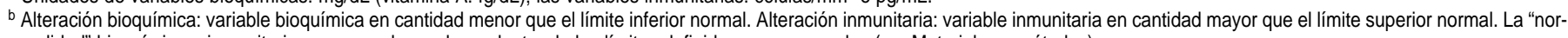
malidad" bioquímica o inmunitaria corresponde a valores dentro de los límites definidos como normales (ver Materiales y métodos).

'En el grupo más afectado no hay casos con valores por debajo del límite inferior normal. Esta situación se aplica para la albúmina, para los linfocitos totales y las subpoblaciones CD3, CD4 y CD8.

d Para hacer la prueba, se usaron los siguientes valores: $\bar{x}=0,1$ y $D E=0,01$. Se aplicó la prueba $\mathrm{F}$ (Epi Info).

de los niños sin la enfermedad, mientras que el riesgo de desnutrición no pareció influir en ello. Cuando se analizó el efecto simultáneo de las dos variables, al comparar los grupos "enfermos con riesgo de desnutrición" y "no enfermos sin riesgo de desnutrición", las únicas diferencias que se apreciaron fueron en apoA1, albúmina, leucocitos, anticuerpos e IL-10, lo que permite afirmar que la influencia del riesgo de desnutrición es insignificante en estos niños. ¿Cómo se puede explicar esto? Pudiera ser que los niños de ambos grupos estuvieran tan afectados por la desnutrición que no fuera posible detectar diferencias en el riesgo de desnutrición entre los dos grupos, por lo que las diferencias apreciadas pudieron deberse solo a la malaria, que produce un empeoramiento de las variables bioquímicas, menores en los niños enfermos, aunque la diferencia no fue significativa.

Los bajos niveles de apoA1 en estos niños parece que no se debieron a diferencias en el estado de nutrición de los dos grupos, sino a la enfermedad que afectaba a uno de ellos, mientras que los mayores antecedentes previos de malaria del grupo que padecía esta enfermedad tampoco contribuyeron a explicar esa diferencia. En efecto, al seleccionar los niños con antecedentes de malaria y analizar los niveles de apoA1 en función de la presencia o ausencia de malaria, se halló diferencia significativa entre los dos grupos, tanto en niños $(66,5 \mathrm{mg} / \mathrm{dL}$ en los enfermos frente a 104,3 mg/dL en los del otro grupo; $t=2,627349, P=0,015383$ ), como en niñas $(75,2 \mathrm{mg} / \mathrm{dL}$ contra $118,0 \mathrm{mg} / \mathrm{dL} ; t=2,595892, P=0,020)$. Luego se demostró que en niños sin antecedentes de malaria, con o sin la enfermedad, los niveles de apoA1 también fueron estadísticamente diferentes en ambos sexos $(P<0,05)$. Finalmente, la idea de que los antecedentes de malaria no contribuyeron a explicar la diferencia en las cifras de apoA1 halladas en estos dos grupos también se demostró cuando, en un primer paso, se seleccionaron los niños con malaria y con antecedentes de malaria y, en un segundo paso, se compararon los valores de apoA1 según el número de episodios de malaria $(1,2$, y 3 o más): los promedios fueron 66,69 y $74 \mathrm{mg} / \mathrm{dL}$, respectivamente, $(\mathrm{F}=$ $0,312 ; P=0,734)$. Así, los únicos rasgos epidemiológicos que difirieron significativamente en los dos grupos - los antecedentes y el número de episodios de malaria- no influyeron en el comportamiento de la apoA1 entre quienes tenían o no malaria en el momento de la investigación. Algo similar ocurrió con los valores medios de prealbúmina e IL-10.

Se debe resaltar que en los dos grupos extremos (con malaria, con riesgo de desnutrición y con alteración bioquímica o inmunitaria, y los que no tenían la enfermedad, ni riesgo de desnutrición, ni alteración bioquímica o inmunitaria), todas las variables bioquímicas e inmunitarias difirieron significativamente, con excepción del título de IgG. La poca edad de la población y, por tanto, el poco tiempo de exposición pueden explicar los bajos títulos de IgG encontrados.

En este grupo de 100 niños y niñas no se halló relación entre los indicadores antropométricos de riesgo de desnutrición y los posibles marcadores 
bioquímicos del mismo, lo cual mantiene la tendencia a usar de modo preferencial los primeros para valorar el estado nutricional.

Uno de los hallazgos más importantes de esta investigación fue la alta frecuencia de valores alterados de IL-10 en los pacientes con malaria, relacionados con la parasitemia pero no con la especie de Plasmodium. Este es el primer trabajo donde se informa este comportamiento de la IL-10 en la infección con $P$. vivax y solo se conocen tres publicaciones relacionadas con la de $P$. falciparum en humanos $(27,42$, 43); sin embargo, la concentración promedio de IL-10 en este trabajo (266 $\mathrm{pg} / \mathrm{mL}$ ) fue mucho más baja que la informada por otros autores, cuyos valores fluctuaron entre $913 \mathrm{pg} / \mathrm{mL}$ (27) en casos de malaria moderada y 2882 $\mathrm{pg} / \mathrm{mL}$ en los casos más graves (44); solo Wenisch y colaboradores (42) encontraron niveles similares a los nuestros $(260 \mathrm{pg} / \mathrm{mL})$. La explicación posible de los bajos niveles encontrados por nosotros puede ser la baja parasitemia de los pacientes.
Agradecimientos. Agradecemos a la Universidad de Antioquia su apoyo económico y financiero; al personal del Hospital de El Bagre y a los niños, sujetos de nuestras preguntas; a la nutricionista Teresita Alzate su participación en el diseño de la encuesta y la recolección de datos de evaluación nutricional; a la inmunóloga Fabiola Toro sus comentarios sobre la parte inmunológica; a los bacteriólogos Marcela Echeverry y Gonzalo Álvarez su ayuda en la recolección de todos los datos y en la realización de la prueba de la gota gruesa.

\section{REFERENCIAS}

1. World Health Organization. Control of Tropical Disease: Malaria Control. Geneva, Switzerland: WHO Office Information; 1995.

2. Servicio Seccional de Salud de Antioquia. Boletín Epidemiológico de Antioquia 1989;11(3): 30-41.

3. Escobar JP. El control descentralizado e integrado de la malaria en Antioquia, Colombia. Biomedica 1997;17(Supl 2):72-75.

4. Galeano LA, Ricaurte CF, Tabares Z, Vélez IM, Jiménez JA, Molina AL, et al. Perfil epidemiológico de Antioquia, 1997. Boletín Epidemiológico de Antioquia 1997;22(3):231-396.

5. Pelletier DL, Frongillo EA, Schroeder DG, Habicht JP. The effects of malnutrition on child mortality in developing countries. Bull World Health Organ 1995;73(4):443-448.

6. Beck MA. Selenium and host defense towards viruses. Proc Nutr Soc 1999;58(3):707-711.

7. Kurpad AV, Jahoor F, Borgonha S, Poulo S, Rekha S, Fjeld CR, et al. A minimally invasive tracer protocol is effective for assessing the response of leucine kinetics and oxidation to vaccination in chronically energy-deficient adult males and children. J Nutr 1999;129(8): 1537-1544.

8. Goodman KJ, Correa P, Tengana Aux HJ, DeLany JP, Collazos T. Nutritional factors and Helicobacter pylori infection in Colombian children. J Pediatr Gastroenterol Nutr 1997;25(5): 507-515.

9. Marcos A. The immune system in eating disorders: an overview. Nutrition 1997;13(10): 853-862.

10. Chandra RK. Nutrition and the immune system. Proc Nutr Soc 1993;52(1):77-84.

11. Chandra RK. Nutrition, immunity and infection: from basic knowledge of dietary manipulation of immune response to practical application of ameliorating suffering and improving survival. Proc Nat Acad Sci USA 1996;93(25):14304-14307.

12. Mc Gregor IA. Malaria: nutritional implications. Rev Infect Dis 1982;4:798-805.
13. Abbas AK, Lichtman AH, Pober JS. Inmunidad frente a los microorganismos. En: Abbas AK, Lichtman AH, Pober JS. Inmunología celular y molecular. Madrid: Interamericana - Mc Graw Hill; 1995. Pp. 369-374.

14. Toro G, Román G, Navarro L. Neurología tropical: aspectos neuropatológicos de la medicina tropical. Santafé de Bogotá: Printer Colombiana; 1983

15. Edirisinghe JS, Fern EB, Targett GA. The influence of dietary protein on the development of malaria. Annals Tropical Paediatrics 1981; 1(2):87-91.

16. Alonso F, Velazco A, Prieto G. Nutrición e inmunidad. Pediatrika 1994;14(10):428-440.

17. Waterlow J. Malnutrición proteicoenergética. Washington D.C.: OPS; 1996. (Publicación Científica 555)

18. Hall JC. Use of internal validity in the construct of an index of under nutrition. J Parenter Enteral Nutr 1990;14(6):582-587.

19. Toure M, Sall NG, Gauthier F, Weill J, Mouray $\mathrm{H}$, Sall M. Apolipoprotein A1 as an early index of protein-energy malnutrition. Eur J Clin Nutr 1991;45(10):511-514.

20. Oska H, Ahonen K, Pasternack A, Marnela KM. Malnutrition in hemodialysis patients. Scand J Urol Nephrol 1991;25(2):157-161.

21. Kremsner PG, Winkler S, Brandts C, Jenne L, Graninger W, Prada J, et al. Prediction of accelerated cure in Plasmodium falciparum malaria by the elevated capacity of tumor necrosis factor production. Am J Trop Med Hyg 1995; 53(5):532-538

22. Nicolas P, Hovette P, Merouze F, Touze JE, Martet G. Cytokines and malaria: a study of TNF-alpha, IL1-beta, IL6 and IL2R in 28 patients. Bull Soc Pathol Exot 1994;87(2): 91-95.

23. Kristensen G, Jacobsen PH. Plasmodium falciparum: characterization of toxin-associated proteins and identification of an hemoglobin containing parasite cytokine stimulator. Exp Parasit 1996;82(2):147-154.
24. Puta C, Hansen MB. Tumour necrosis factor alpha in uncomplicated malaria in young adults. Trop Geogr Med 1995;47(3):134-135.

25. Yamada-Tanaka MS, Ferreira Da Cruz MF, Alecrim MG, Mascarenhas LA, Daniel-Ribeiro CT. Tumor necrosis factor alpha, interferon gamma and macrophage stimulating factor in relation to the severity of Plasmodium falciparum malaria in the Brazilian Amazon. Trop Geogr Med 1995;47(6):282-285.

26. Nyakundi JN, Warn P, Newton C, Mumo J, Jephthah-Ochola J. Serum tumour necrosis factor in children suffering from Plasmodium falciparum infection in Kilifi District, Kenya. Trans R Soc Trop Med Hyg 1994;88(6): 667-670.

27. Schwarz M, Majdic O, Knapp W, Holter W. High-level IL-10 production by monoclonal antibody-stimulated human T cells. Immunology 1995;86(3):364-371.

28. Wang P, Wu P, Siegel MI, Egan RW, Billah MM. Interleukin (IL-10) inhibits nuclear factor kappa B (NF kappa B) activation in human monocytes. IL-10 and IL-4 supress cytokine synthesis by different mechanisms. J Biol Chem 1995;270(16):9558-9563.

29. Centers for Disease Control and Prevention (CDC), USA; World Health Organization (WHO), Geneva, Switzerland. Epi Info 6. Un procesador de textos, base de datos y estadísticas para la salud pública. Versión 6.04a. Atlanta (USA): CDC y WHO; julio de 1996.

30. WHO Working Group. Use and interpretation of anthropometric indicators of nutritional status. Bull World Health Organ 1986;64: 929941

31. Gorstein J, Sullivan K, Yip R, de Onis M, Trowbridge F, Fajans P, Clugston G. Issues in the assessment of nutritional status using anthropometry. Bull World Health Organ 1994;72(2):273-283.

32. Hijmans W, Schmit HRE, Jongsma APM, Ploem JS. Performance testing of fluorescent antisera against human immunoglobulins. En: Holborow EJ (editor). Standardization in 
immunofluorescence. Oxford and Edinburgh: Blackwell Scientific Publications; 1970. Pp. 193202.

33. Lambros C, Vanderberg JP. Synchronization of Plasmodium falciparum erythrocytic stages in culture. J Parasitol 1979;65(3):418-420.

34. Commans-Bitter WM, de Groot $\mathrm{R}$, van den Beemd R, Neijens HJ, Hop WCJ, Groeneveld $\mathrm{K}$, et al. Immunophenotyping of blood lymphocytes in childhood. J Pediatrics 1997;130(3): 388-393.

35. Stites DP. Métodos de laboratorio clínico para detección de inmunidad celular. En: Stites DP, Terr AI, Parslow TG. Inmunología básica y clínica. 8. ${ }^{a}$ ed. México DF: El Manual Moderno; 1996. Pp. 256-260.

36. López-Antuñano FJ, Schmunis G, eds. Diagnóstico de malaria. Washington D.C.: OPS; 1988. (Publicación Científica 512).

37. Statgraphics Plus: Statistical graphics system [programa para computador]. Manugistec versión 7.1. Estados Unidos: Statistical Graphics Corporation; 1993.
38. Ya-lun Chou. Análisis estadístico. México DF: Editorial Interamericana; 1972.

39. Feillet F, Parra HJ, Kamian K, Bard JM, Fruchart JC, Vidailhet M. Lipoprotein metabolism in marasmic children of Northern Mauritania. Am J Clin Nutr 1993;58:484-488.

40. Organización Mundial de la Salud, Oficina Sanitaria Panamericana, Programa de atención integrada de las enfermedades prevalentes de la infancia (AIEPI). Investigaciones operativas de AIEPI. Versión preliminar. Washington D.C.: OPS; 2000.

41. Shankar AH. Nutritional modulation of malaria morbidity and mortality. J Infect Dis 2000;182(Suppl 1):S37-S53.

42. Wenisch C, Parschalk B, Narzr E, Looareesuwan S, Graninger W. Elevated serum levels of IL-10 and INF gamma in patients with acute Plasmodium falciparum malaria. Clin Immunol Immunopathol 1995;74(1):115-117.

43. Ho M, Sexton MM, Tongtawe P, Looareesuwan S, Suntharasama P, Webster HK. Interleukin-10 inhibits tumor necrosis factor production but not antigen specific lymphoproliferation in acute Plasmodium falciparum malaria. J Infect Dis 1995;172(3):838-844.

44. Allan RJ, Rowe A, Kwiatkowski D. Plasmodium falciparum varies in its ability to induce tumor necrosis factor. Infect Immun 1993; 61(11):4772-4776.

Manuscrito recibido el 23 de febrero de 2001 y aceptado para publicación, tras revisión, el 17 de octubre de 2001.

ABSTRACT Objective. To explore some relationships among immunity, nutrition, and malaria in a group of children from a population with a high endemic incidence of malaria.

Methods. This pilot descriptive prospective cross-sectional study was carried out in

Malaria in children: links between nutrition 1998 in the municipality of El Bagre, Antioquia, Colombia. Both boys and girls were studied in two groups: one group of 51 children had malaria and another group of 49 did not have malaria. The children with malaria had symptoms or signs of malaria and a positive thick blood film. The comparison group of 49 children without malaria (no signs or symptoms of malaria and a negative thick blood film) were chosen at random at government schools or children's centers. For the study the following indices were calculated: weight-for-age, height-for-age, and weight-for-height. Also measured were the blood-serum concentrations of: albumin, prealbumin, apolipoprotein A1 (apoA1), transferrin, zinc, vitamin A, immunoglobulins G and M, interleukin-10 (IL-10), tumor necrosis factor-alpha, interferon-gamma, and lymphocyte populations.

Results. Of the children studied, $69 \%$ presented some risk of malnutrition, and $63 \%$ had some risk of chronic malnutrition. With regard to the immunity and biochemical variables, the children with malaria had less apoA1 and albumin and more IL-10 than did the children without malaria. All the biochemical variables showed lower averages in the group with malaria, risk of malnutrition, and immune or biochemical changes, while all the immunity variables had higher averages in that same group of children.

Conclusions. 1) The high frequency of chronic malnutrition found clearly indicates the need for food supplementation measures. 2) The low values of prealbumin found could be due to the frequent bacterial or viral infections reported. 3) The serious vitamin A deficiency found calls for a supplementation program. 4) An association was found between low apoA1 values and the presence of malaria, but which one follows from the other is not known. 5) No relationship was observed between the anthropometric indicators of risk of malnutrition and the possible biochemical markers of malnutrition. 6) We found high levels of IL-10 in the children with malaria; this is the first time that this has been reported for Plasmodium vivax. 